\title{
Analysis on Mars Exploration for the Prospect of Future Mars Migration Plan and Society Development
}

\author{
Yueyang Zhang ${ }^{1, *}$ \\ ${ }^{1}$ The University of Manchester, Manchester, United Kingdom, M1 7FU \\ *Corresponding author. Email: yueyang.zhang@student.manchester.ac.uk
}

\begin{abstract}
Mars is the planet which is the closest to the earth in the solar system, humans have never stopped exploring its topography, soil characteristics and resource composition. Different from the earth-moon exploration, there are still some difficulties in the exploration of Mars. The planet's orbital period of the earth and Mars is different, so the mission available period is limited, the distance of the ground fire is between 56 trillion and 400 million, so there is weaker solar radiation and thinner atmosphere. The long-distance journey will not only increase the delay of radio transmission and lengthen the exploration period, but also make the human mission to Mars need to consider the consumption of resources and other issues. This paper will introduce the differences and difficulties between Mars exploration and Earth-moon exploration from the motion law, distance of Earth to Mars, solar radiation and atmospheric environment. It focuses on the study of geological structure, soil and water ice, material composition and environment. It also list the lack and difficulty in data, long-distance travel and continually supply for Mars exploration. Finally, this paper analyzes and integrates Mars-related materials to provide theoretical guidance and solutions for future Mars exploration and immigration.
\end{abstract}

Keywords: exploration of Mars, Mars migration program, Martian surface

\section{INTRODUCTION}

With the development of science and technology, human space exploration has never stopped. As the planet in the solar system the closest to the earth's environment [1], Mars has always been the primary detection target for human beings for exploration and immigration. Since the discovery of the Mars Canal in Italy, 1877, [2] it has provided evidence for the existence of liquid water and life, humans have also begun to think about the possibility of emigrating to Mars. This paper will start with the introduction of the Martian environment to help understand the process of modern Mars exploration, and the future manned Mars plan Facing problems and prospects for the construction of Mars society in the future.

Nowadays, human beings have already sent planetary probe on Mars, so the problem is how can scientists send people on Mars, finally send immigrant to mars and ensure their long-term survival. From current exploration and data, scientists can have the basic concept of resources on Mars, this paper will summarize Mars exploration activity, analyse the difficulty people faced and show the plan of Mar migration.

In the context of population expansion, this paper analyzes and integrates Mars-related materials to provide theoretical guidance and solutions for future Mars exploration and immigration.

\section{DIFFICULTIES IN MARS EXPLORATION}

Since the 1960s, the United States and the Soviet Union have been competing in the field of Mars exploration. Up to now, many countries in the world are committed to the development of Mars exploration. Until September 2021, there are 49 Mar-exploration missions. Among them, the United States NASA 23 times, Soviet Union/Russia 20 times, Japan ISAS 1time, European Space Agency, ESA 2 times, India ISRO 1-time, United Arab MBRSC 1 time, China CNSA 1 time. Obviously, Mars is a planet in the solar system that is closer to the earth and has a natural environment like that of the earth. The exploration of Mars has always been of great significance. 
In these terms of period, the exploration process of Mars can be divided into three stages. In the first stage (1960-1975), 23 missions had been implemented. The main feature is because of the background of the Cold War that the United States and the Soviet Union want to demonstrate their national compete capabilities. In the second stage (1976-1990), with the focus shift of the US-Soviet competition strategy, Mars exploration entered a trough, and only two missions were implemented [3]. In the third stage (from the 1990s to the present), 24 missions were implemented, with the development of new technologies and scientific discoveries as the main driving force, which gave birth to another peak of Mar's exploration. Excluding the MBRSC's Emirates Mars Mission and NASA's Mars 2020 which still in progress, there are 25 successful missions, successful accounting for about $53 \%$.

Different from Earth-Moon exploration, the difficulty of Mars exploration is not only in the distance, environmental differences, and differences in relative motion of Earth and Mars, which have brought qualitative changes to Mars exploration missions. These differences are mainly reflected in the following aspects.

1. The difference of the planet's orbital period. Compared to Mars, the distance between Moon and the Earth are closer. Mars, same as earth, revolve around the sun with the earth under the gravitational force of the sun. In this situation, Mars exploration only happens once every 26 months [3].

2. The distance between Mars and Earth is about 56 million $\sim 400$ million $\mathrm{km} \mathrm{[3].} \mathrm{The} \mathrm{farthest} \mathrm{distance} \mathrm{is}$ about 1,000 times the distance between the Earth and the Moon, and the radio signal transmission delay reaches $23 \mathrm{~min}$ [3]. This difference needs higher requirements for the space-to-earth measurement and control communication capability and the autonomous operation capability of the detector.

3. Solar radiation is weaker, the average distance between the earth and the moon from the sun is about 150 million $\mathrm{km}$, the average solar constant is about $1,378 \mathrm{~W} / \mathrm{m}^{2}$, the average distance between Mars and the sun is about 230 million $\mathrm{km}$, and the average radiation intensity is about $589 \mathrm{~W} / \mathrm{m}^{2} .43 \%$ [3]. It means that when people put solar panel, the efficiency is much less than on the Moon. That difference put higher requirements for the detector conversion efficiency.

4. Mars has a thin atmosphere. The surface of the moon is approximately vacuum, and the surface of the Mars has an atmosphere with a density of about $1 \%$ of the earth, include $96 \%$ carbon dioxide and less than $0.2 \%$ oxide and there are strong winds and dusty weather $[3,4]$. The influence of the atmosphere makes it more difficult to land on Mars.

5. The lowest temperature on Mars is minus 133 Celsius, usually minus 63 Celsius [4]. Although Mars is the closest to the Earth's temperature among the eight planets in the solar system, the temperature is still harsh. Currently, space equipment can land normally, but it needs to be improved as a place for human long-term survival.

\subsection{The Environment of Mars}

On Mars, there are many impact craters and large impact basins, shield volcanoes, aeolian dunes, Martian polar ice caps, canyon systems, dry riverbeds, and ditches on the surface of Mars. The extensive development of dry riverbeds, deltas, alluvial fans, and ditches reflect the existence of liquid water on the surface of Mars. These flowing water landforms record the continuous erosion and transformation of the Martian surface. On the earth, the existence of water environment is often related to life activities. Therefore, one of the key objectives of various Mars exploration missions always includes exploring and tracking the different forms and evidence of water used or currently on the surface of Mars, such as polar caps, permanent water ice, ground water ice, short-term liquid water on the surface, and water that exists in minerals in different forms $[5,6]$.

The water-based system is the basic requirement for the life born and sustain life. So the type and abundance of water-bearing minerals provide information about the temperature, salinity, and $\mathrm{pH}$ of the aqueous solution when these minerals were formed.

Without liquid water and vegetation cover, there are a lot of loose sediments on the surface of Mars, which is a planet surrounded by dust [7]. Which creates the suitable condition for the formation of aeolian landforms. Compared with the sand which is rich in quartz on Earth, Martian aeolian sand dominated by basalt [8].

\subsection{Exploration of Mars}

The purposes to explore Mars is basically for the following purposes.

1. Study the morphology and geological structure characteristics of Mars. Detect the global topography and geomorphology characteristics of Mars, obtain high-precision topographic data of typical regions, and conduct research on the genesis and evolution of Martian geological structures.

2. Study the soil characteristics and water ice distribution on the surface of Mars. Detect Martian soil types, weathering and sedimentary characteristics and global distribution, search for water ice information, and conduct research on the layered structure of Martian soil profiles. 
3. Study the composition of element on the surface of Mars. Identify the types of rocks on the surface of Mars, explore secondary minerals on the surface of Mars, and operate surface mineral composition analysis.

4. Study the atmospheric ionosphere and surface climate and environmental characteristics of Mars. Detect the Martian space environment and the surface temperature, pressure, and wind field of Mars, and do research on the Martian ionospheric structure and seasonal changes in surface weather.

5. Study the physical field and internal structure of Mars. Detect the magnetic field characteristics of Mars. Carry out research on the early geological evolution history of Mars and the internal mass distribution and gravity field of Mars.

\section{THE PROCESS AND FUTURE PROSPECT}

With the background of Mars, scientists processed a series of work on Mars exploration, but still have many problems to solve on different aspect. This part will talk about the difficulties people are confronted with and the way to solve, in the last part, the plan of Martian immigration will give step by step.

\subsection{Lack of data on Mars}

Up to now, although many Mars exploration missions have been successfully implemented, the current understanding of Mars space (environmental gravitational field, magnetic field, atmosphere, etc.) is still inadequate, and the exploration success rate is not particularly high, so it still needs to do more experiment, and earn extra experience, make sure for the future exploration.

\subsection{Power aspect}

Modern rocket power systems have solid and liquid fuel propulsion, as well as nuclear reaction and solar propulsion. The spacecraft can use the reaction force of fuel propulsion to provide the initial speed and direction. Because there is no resistance in the universe. According to Newton's first law, the spacecraft can keep travel without continuous power.

\subsection{Travel aspects}

Due to the long distance of the Earth and Mars and the reasons of gravitation and Mars orbital revolution, we should adopt the course trajectory of Hohmann's transfer orbit (dotted line) instead of the shortest distance between the earth and Mars (as shown in Figure 1). What we expect is let Mar and spacecraft contact at point $\mathrm{A}$.

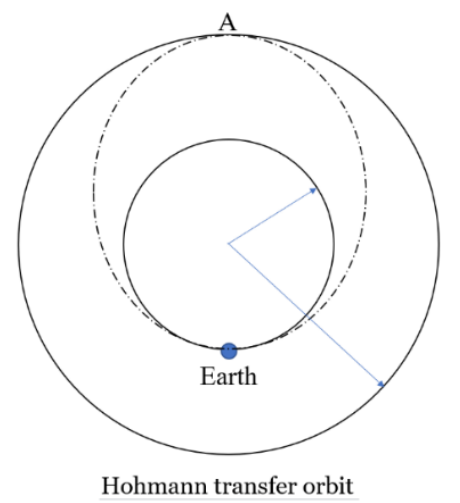

Figure 1 Hohmann transfer orbit

According to Kepler's second law, the speed at the perihelion is the largest and the speed at the aphelion is the smallest. When the spacecraft flies to the position shown in the figure, the speed is small and the required deceleration is small, which is an ideal solution.

\subsection{Manned spaceflight and Martian immigration}

In the case of short-term investigations, scientists can use the design of adding a working space capsule when launching the spacecraft to ensure short-term living resources and working instruments for astronauts on Mars. This kind of investigation is suitable for sample collection.

For long-term exploration, scientists can use the plan of launching multiple rockets and spacecraft at the same time, astronaut stay on one of them, and the remaining rockets are prepared to take resources for long-term survival to prepare for long-term life and do experiment and obtain evidence.

According to surveys, it has been shown that Mars once had enough internal energy [9]. In the case of longterm survival work, when the astronauts need additional survival resources on Mars, electricity can be supplemented in the form of geothermal power generation and solar power generation. At present, the surface of Mars is in an arid and cold world, without liquid water, and the atmosphere is mainly carbon dioxide [10, 11]. Tectonic and magmatic activities have basically ceased, and the water may be transferred to the underground [12, 13]; therefore, look for underground frozen water resources. It is also one of the key points of exploration.

Compared with the aeolian sand on Earth, which is rich in quartz, Martian aeolian sand is dominated by basalt particles, while in parts of the Arctic desert it is gypsum particles. After finding water resources, improving the soil suitable for crop growth can also 
transform the environment of Mars. For oxygen, the oxygen used in space flight in the past was supplied by liquid oxygen, but to prevent suffocation, the percentage of carbon dioxide must be controlled to a balance. The more mature method currently used is to use superoxide to remove carbon dioxide by adding granular anhydrous LiOH to help remove part of the carbon dioxide. However, the long-term method of synthesizing oxygen is still needed to study.

After experiments, if the long-term survival plan of mankind can be implemented, the Martian immigration can be considered. To ensure the development of human food, clothing, house construction, and transportation, it is extremely important to design the source of Martian immigration and the social structure.

Because the initial number of immigrants is limited, it is impossible to bring a large amount of survival resources to Mars. This plan must be implemented when the Mars survival plan is quite complete. At the same time, immigrants can be self-sufficient through resources on Mars.

To build a Martian society, the early stage of immigration needs to rely on highly educated researchers to further transform the living environment of Mars [3]. Among them, for long-term survival, agriculture and industry are essential to provide a stable environment for immigrant life. Long-term survival also requires the construction of roads and houses. Public infrastructure and housing can be constructed through the development of the construction industry. Finally, the Martian immigration plan is an industry that needs long-term development. To maintain social stability and public security and harmony, there should also be people engaged in the service industry in the Martian society.

\section{CONCLUSION}

Mars exploration is still a long-term plan. There are still many unknow part about Mars. Scientist still face the problem of long-distance travel and different planet's orbital period, Hohmann's transfer orbit is the best choice now. From the exploration data, Mars does not have water on the surface but may transfer to the underground. The purpose of Mars exploration is to know the physical structure, the resource distribution, even though people cannot take too much resource to Mars, exploring the underground water and soil improvement have to be done before the immigration. The significance of this paper is to understand the history and recent progress of Mars exploration by summarizing materials, to make the difficulties faced in Mars exploration and the planning steps for future immigration clearer and more logical.

The space exploration still faces many challenges, such as long-term method of synthesizing oxygen, therefore, the development of aviation requires not only the efforts of aerospace scientists, but also the development of other industries (such as chemistry), which require the unremitting efforts of both scientist and engineer. Overcome the difficulties, people should start with further manned Mars exploration, and finally realize the Mars migration plan based on sociological analysis.

\section{AUTHORS' CONTRIBUTIONS}

This paper is independently completed by Yueyang Zhang.

\section{ACKNOWLEDGMENTS}

First of all, my deepest gratitude goes to Professor Ian Eames, with his guidance and teaching, he has walked me through all the stages of the writing of the thesis and helped me a lot in knowledge. Without his teaching, this thesis could not have reached its present form. I am also greatly indebted to my supervisor Ms. Cuihong Wang and Dr. May Hu, who have instructed me about the structure of thesis, supported me about the professional knowledge and given me good suggestion. Finally, I would show my thanks to my beloved family for their support and encouragement.

\section{REFERENCES}

[1] DAVID S F P. Humans to Mars: fifty years of mission planning, 1950-2000, NASA Monographs in Aerospace History Series, Available as NASA SP-2001-4521 [R] USA: NASA, 2001.

[2] KENNETH C. NASA Mars 2020 rover gets a landing site: a crater that contained a lake - the rover will search the Jezero Crater and delta for the chemical building blocks of life and other signs of past microbes [N] The New York Times, 2018-1119.

[3] GENG Y, ZHOU J S, LI S, et al. Review of first Mars exploration mission in China [J] Journal of Deep Space Exploration, 2018, 5(5): 399-405.

[4] Ruxue L, Dan L, et al. A Class of Two Dimensional Dynamic Model with Time Delays Describing Environment Pollution and Its Stability Analysis [J] Mathematical modelling and application, 2017, 6(2).

[5]Hubbard G S, Naderi F M and Garvin J B. 2002. Foll owing the water, the new program for Mars exploration. Acta Astronautica, 51(1-9): 337-350.

[6] Gou S, Yue Z Y, Di K C and Zhang X. Advances in aqueous minerals detection on Martian surface. Journal of Remote Sensing, 2017, 21(4): 531-548. 
[7] Antoniadi E M. The Planet Mars[M]. Shaldon Devon, UK; Keith Reid Limited, 1975: 1-65.

[8] Jiyan L, Zhibao D, Research Progress of Acolian Landforms on Mars [J] Journal of desert research 2016, 7(4).

[9] VIOREL, B. Mars: prospective energy and material resources (illustrated ed.) [M] Germany: Springer Science \& Business Media, 2009.

[10] WILLIAMS, D. R. Mars fact sheet [Z] USA: National Space Science Data Center, NASA, 2010.

[11] MAHAFFY, P. R, WEBSTER C R, ATREYA S K, et al. Abundance and isotopic composition of gases in the martian atmosphere from the Curiosity Rover [J] Sciencemag.org., 2013, 341 (6143):263266.

[12] WEBSTER, G, BROWN D. Curiosity Mars Rover sees trend in water presence [Z] USA: NASA, 2013.

[13] Mcewen, A, LUJENDRA, O, DUNDAS, C., et al. Seasonal flows on warm martian slopes [J] Science, 2011, 333(6043): 740-3. 